\title{
38.2-Gb/s Optical-Wireless Transmission in 75-110 GHz Based on Electrical OFDM with Optical Comb Expansion
}

Deng, Lei; Pang, Xiaodan; Beltrán, Marta; Zhang, Xu; Arlunno, Valeria; Zhao, Ying; Yu, Xianbin; Llorente, Roberto; Liu, Deming; Tafur Monroy, Idelfonso

Published in:

OFC/NFOEC Technical Digest

Publication date:

2012

Document Version

Publisher's PDF, also known as Version of record

Link back to DTU Orbit

Citation (APA):

Deng, L., Pang, X., Beltrán, M., Zhang, X., Arlunno, V., Zhao, Y., Yu, X., Llorente, R., Liu, D., \& Tafur Monroy, I. (2012). 38.2-Gb/s Optical-Wireless Transmission in 75-110 GHz Based on Electrical OFDM with Optical Comb Expansion. In OFC/NFOEC Technical Digest Optical Society of America.

\section{General rights}

Copyright and moral rights for the publications made accessible in the public portal are retained by the authors and/or other copyright owners and it is a condition of accessing publications that users recognise and abide by the legal requirements associated with these rights.

- Users may download and print one copy of any publication from the public portal for the purpose of private study or research.

- You may not further distribute the material or use it for any profit-making activity or commercial gain

- You may freely distribute the URL identifying the publication in the public portal 


\title{
38.2-Gb/s Optical-Wireless Transmission in 75-110 GHz Based on Electrical OFDM with Optical Comb Expansion
}

\author{
Marta Beltrán ${ }^{(1)}$, Lei Deng ${ }^{(2)(3)}$, Xiaodan Pang ${ }^{(2)}$, Xu Zhang $^{(2)}$, Valeria Arlunno ${ }^{(2)}$, Ying Zhao ${ }^{(2)}$, \\ Xianbin Yu ${ }^{(2)}$, Roberto Llorente ${ }^{(1)}$, Deming Liu ${ }^{(3)}$, and Idelfonso Tafur Monroy ${ }^{(2)}$ \\ (1) Valencia Nanophotonics Technology Center, Universidad Politécnica de Valencia, Camino de Vera s/n, 46022 Valencia, Spain \\ (2) DTU Fotonik, Department of Photonics Engineering, Technical University of Denmark, 2800 Kgs. Lyngby, Denmark \\ (3) College of Optoelectronics Science and Engineering, HuaZhong University of Science and Technology, 430074 Wuhan, China \\ mbeltran@ntc.upv.es
}

\begin{abstract}
We demonstrate scalable optical comb- and heterodyning-based generation, optical and $1.3-\mathrm{m}$ wireless transmission, and electrical heterodyne detection of multiband OFDM up to $38.2 \mathrm{~Gb} / \mathrm{s}$ occupying 14.4-GHz RF bandwidth, for high-capacity optical-wireless links in $75-110 \mathrm{GHz}$.

OCIS codes: (060.2330) Fiber optics communications; (060.5625) Radio frequency photonics
\end{abstract}

\section{Introduction}

Millimeter-wave wireless systems are a potential solution for future seamless integrated optical/wireless access as well as for mobile backhauling [1]. Millimeter-wave bands at around $60 \mathrm{GHz}$ and higher could provide bandwidth enough to easily support multi-gigabit capacities. The $60-\mathrm{GHz}$ band has been widely studied as it provides about 7-GHz bandwidth for unlicensed communications [1,2]. Bands of around $100 \mathrm{GHz}$ and higher show larger potential bandwidths available to support very high capacities envisioned (towards $100 \mathrm{~Gb} / \mathrm{s}$ ) [3]. In particular, the 75-110 GHz band (W-band) can extend outdoor transmission distances due to its lower atmospheric loss. Radio-over-fiber systems in the $75-110 \mathrm{GHz}$ band are recently attracting more and more interest to deliver $40 \mathrm{~Gb} / \mathrm{s}$ and beyond [1, 4-7]. Wireless transmission systems in the $75-110 \mathrm{GHz}$ band have been reported up to 20 Gb/s [4] based on ASK data modulation. Spectral efficient modulation formats have also been employed, up to $3 \mathrm{~cm}$ of wireless distance at $20 \mathrm{~Gb} / \mathrm{s}$ based on QPSK [5] or at $40 \mathrm{~Gb} / \mathrm{s}$ based on 16-QAM [6]. A system based on optical OFDM with optical down-conversion has also been demonstrated without wireless transmission [7].

In this paper, we report on the optical generation, combined optical and 1.3-m wireless transmission, and electrical detection in the $75-110 \mathrm{GHz}$ band of electrical OFDM based on 16-QAM subcarrier modulation up to $38.16 \mathrm{~Gb} / \mathrm{s}$ occupying a RF bandwidth of $14.414 \mathrm{GHz}$, with a bit error rate (BER) performance within the standard forward error correction (FEC) limit of $2 \cdot 10^{-3}$. The proposed system has the following advantages: 1) Electrical OFDM with a high number of subcarriers has not been reported in the $75-110 \mathrm{GHz}$ band to our knowledge although it has been widely used in wireless systems to benefit from its high spectral efficiency, flexibility, and robustness to fiber impairments and wireless multipath fading; 2) RF bandwidth seamless scalability is demonstrated enabled by optical comb generation; 3) Optical heterodyne up-conversion avoids the need for high-frequency electro-optical devices and provides transparency to the RF carrier frequency [7].

\section{Experimental setup}

Figure 1 shows the schematic of the experimental setup. At the optical OFDM transmitter, a baseband OFDM signal is generated by offline digital signal processing (DSP), which is then stored in an arbitrary waveform generator (Tektronix AWG7122C). In the offline DSP, a data stream consisting of a pseudo-random bit sequence (PRBS) of length $2^{15}-1$ is mapped onto 72 16-QAM subcarriers, which, together with 8 pilot subcarriers, one zero-power DC subcarrier, and 47 zero-power edge subcarriers, are converted to the time domain via an IFFT of size 128. A cyclic prefix of length 13 samples is employed, resulting in an OFDM symbol size of 141. To facilitate OFDM frame synchronization and channel estimation, 10 training symbols are inserted at the beginning of each OFDM frame that contains 150 data symbols. The real and imaginary parts of the OFDM signal are clipped and converted to analog signals by two D/A converters operating at $5 \mathrm{GS} / \mathrm{s}$ with a D/A resolution of 10 bits. The two analog signals from the AWG are filtered by two antialiasing low-pass filters (LPF) with 2.5-GHz bandwidth. The two filtered signals are amplified and applied to an optical I/Q modulator, which is biased at the minimum transmission point, connected to an external-cavity laser (ECL, $\lambda_{1}=1550.6 \mathrm{~nm}$ ) with $100-\mathrm{kHz}$ linewidth. An optical OFDM signal at $9.57 \mathrm{~Gb} / \mathrm{s}$ $(5 \mathrm{~Gb} / \mathrm{s} \cdot 4 \cdot 72 / 141 \cdot 150 / 160)$ with a bandwidth of $3.164 \mathrm{GHz}(5 \mathrm{GHz} \cdot 81 / 128)$ is thus generated. An Erbium-doped fiber amplifier (EDFA) is employed to compensate for loss and an optical band-pass filter with 0.8-nm bandwidth is employed to filter out-of-band noise. Subsequently, the optical OFDM signal is expanded by a comb generator based on an overdriven phase modulator (PM) [8] to form a five-band 47.85-Gb/s signal. The generated comb is 


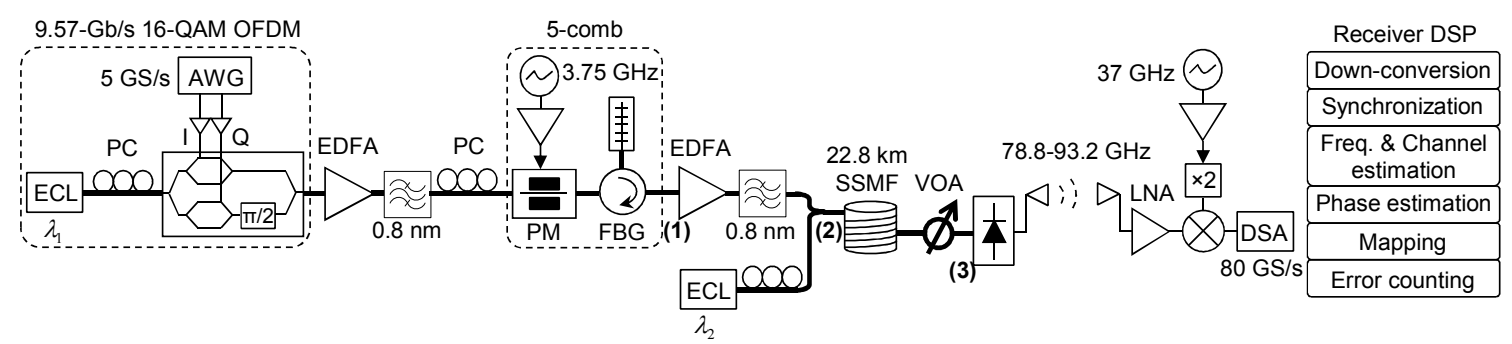

Fig. 1. Experimental setup of a hybrid wireless-optical system in the $75-110 \mathrm{GHz}$ band based on electrical OFDM with optical comb expansion. PC: Polarization controller. VOA: Variable optical attenuator.
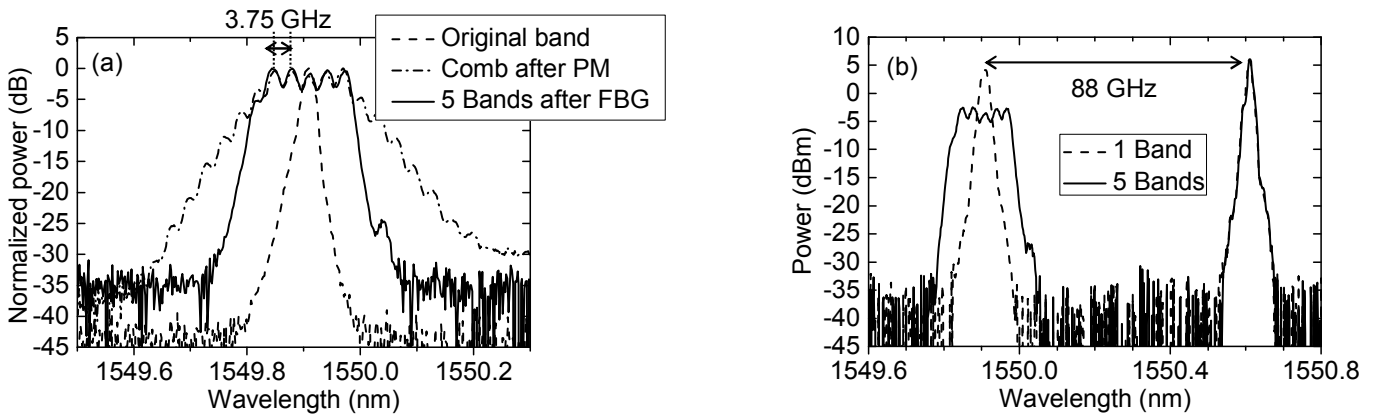

Fig. 2. (a) Optical spectra measured at various points in Fig. 1. (b) Optical spectra measured at point (2) in Fig. 1. (Resolution: $0.01 \mathrm{~nm}$ )

filtered by a 25-GHz fiber Bragg grating (FBG) filter operating in reflection, as shown in Fig. 2(a), to reduce crosstalk penalty. The frequency spacing of the comb lines is set to $3.75 \mathrm{GHz}$ to minimize crosstalk penalty while maximizing spectral efficiency. After EDFA amplification and noise filtering, the optical five-band OFDM signal at point (1) in Fig. 1 is amplified and combined with an unmodulated CW optical carrier (ECL, $\left.\lambda_{2}=1549.9 \mathrm{~nm}\right)$ with $100-\mathrm{kHz}$ linewidth. The combined optical five-band OFDM signal and the unmodulated CW carrier would be transmitted to a remote antenna site where they are heterodyne mixed in a 100-GHz photodetector ( $\mathrm{u}^{2} \mathrm{t}$ XPDV4120R). Optical transmission over $22.8 \mathrm{~km}$ of standard single-mode fiber (SSMF) is evaluated in the experiment. The signal after photodetection is the electrical OFDM signal at the desired RF carrier frequency, which is fed to a rectangular horn antenna in the $75-110 \mathrm{GHz}$ band with 24-dBi gain. Fig. 2(b) shows the spectrum of the combined optical signal at point (2) in Fig. 1, the RF carrier frequency is set to $88 \mathrm{GHz}$. After wireless transmission, the RF OFDM signal is received by another rectangular horn antenna in the 75-110 GHz band with 25-dBi gain. The received signal is amplified by a low-noise amplifier (LNA, Radiometer Physics 75-105 GHz) with 25-dB gain. Frequency down-conversion of the RF OFDM signal is performed by electrical mixing $(75-110 \mathrm{GHz}$ RF, 1-36 GHz IF) with a local oscillator (LO) signal at $74 \mathrm{GHz}$. The LO signal is generated by frequency doubling a 37-GHz signal from a signal generator (Rohde\&Schwarz SMF100A). The down-converted signal is sampled at $80 \mathrm{GS} / \mathrm{s}$ by a digital signal analyzer with $32-\mathrm{GHz}$ real-time bandwidth (Agilent DSAX93204A) and demodulated by offline DSP. The receiver-side DSP blocks are shown in Fig. 1. In the receiver DSP, first, frequency down-conversion and LPF is performed to extract each baseband OFDM band. For each baseband OFDM band, synchronization, frequency and channel estimation, pilot-assisted phase estimation, data recovery by symbol mapping and serialization, and bit error rate (BER) test is performed. To eliminate the dispersion and nonlinearity effects induced by fiber and wireless transmission, one-tap equalizer and an effective algorithm combining intra-symbol frequency-domain averaging [9] and digital phase-locked loop are used for channel estimation. BER is evaluated by counting the number of errors considering 42912 bits.

\section{Experimental results}

Up to 4 RF OFDM bands out of the 5 optical OFDM bands can be demodulated within the FEC limits due to the frequency response of the photodetector. Fig. 3 shows the electrical spectra of the single-band and four-band OFDM signals after sampling at the receiver. BER performance of the four-band OFDM signal at $38.16 \mathrm{~Gb} / \mathrm{s}$ has been evaluated and compared with the performance of the 9.57-Gb/s single-band OFDM signal without comb expansion, i.e. when the RF signal driving the PM is off. Examples of recovered constellations are also shown in Fig. 3. Fig. 4 shows the measured BER as a function of the received optical power at point (3) in Fig. 1. Fig. 4(a) shows BER for combined optical and wireless transmission of the single-band OFDM signal. The receiver sensitivity at the FEC 

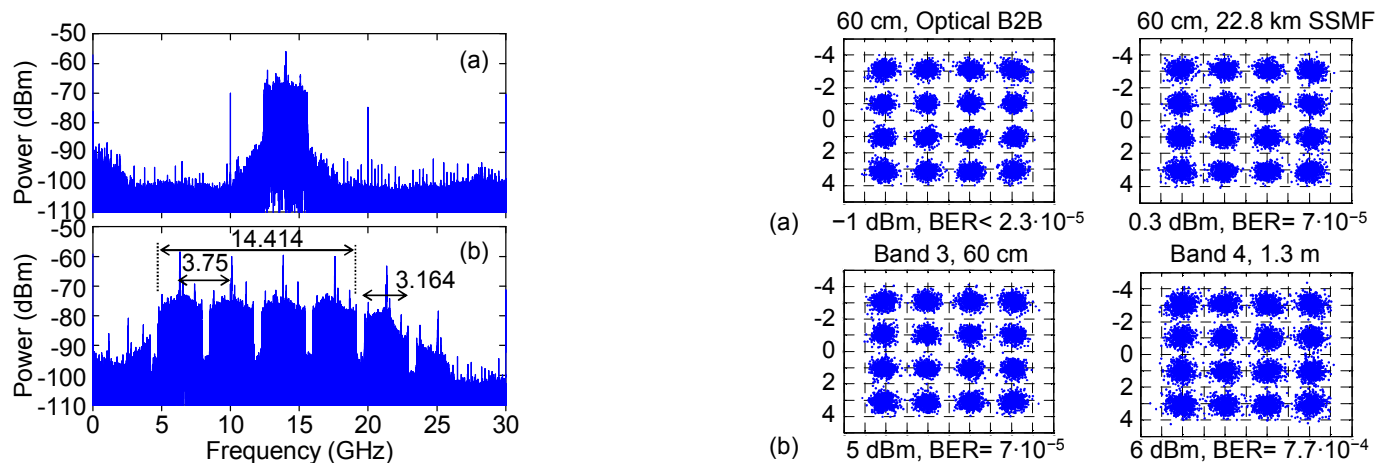

Fig. 3.Electrical spectra after sampling at the receiver at 4.5-dBm received optical power for optical B2B and 60-cm wireless distance, and recovered constellations. (a) 9.57-Gb/s single-band OFDM signal. (b) 38.16-Gb/s four-band OFDM signal.
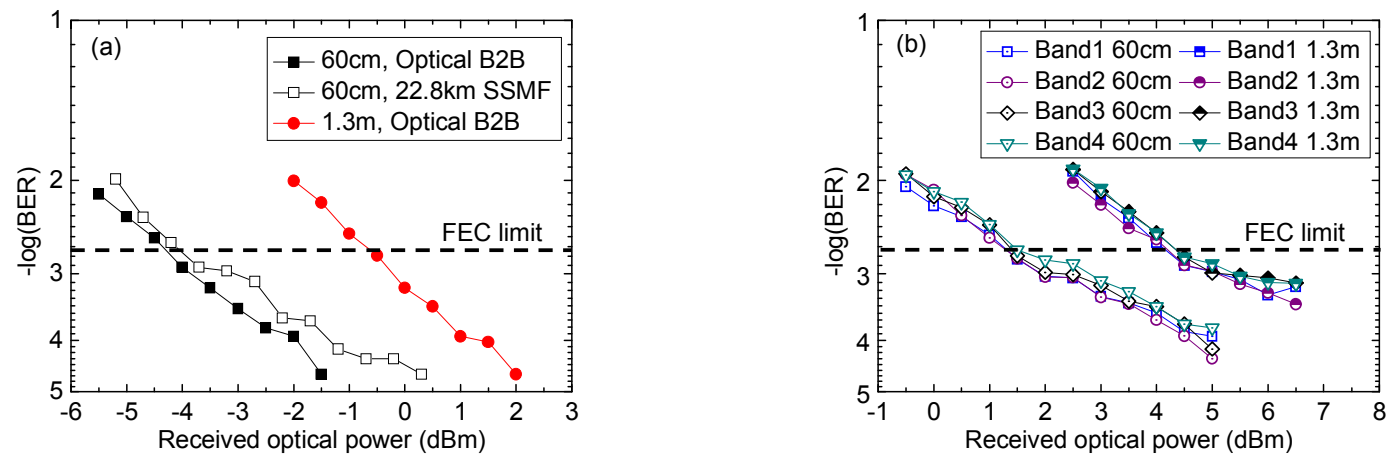

Fig. 4.BER performance as a function of received optical power. (a) 9.57-Gb/s single-band OFDM signal as a function of wireless-optical transmission distance. (b) 38.16-Gb/s four-band OFDM signal as a function of wireless distance for optical B2B.

limit of $2 \cdot 10^{-3}$ is $-4.4 \mathrm{dBm}$ and $-0.7 \mathrm{dBm}$ for optical back-to-back (B2B) and $60 \mathrm{~cm}$ and $1.3 \mathrm{~m}$ of wireless distance, respectively. Optical transmission over $22.8 \mathrm{~km}$ of SSMF induces $0.3 \mathrm{~dB}$ receiver sensitivity penalty. BER is degraded for received optical power higher than $0.3 \mathrm{dBm}$ due to fiber nonlinearity, corresponding to an optical power of $5.8 \mathrm{dBm}$ at the input of fiber. Fig. 4(b) shows the wireless transmission performance of the four-band OFDM signal for optical B2B. There is negligible power penalty among the different OFDM bands when one OFDM subcarrier in the second band is removed during BER evaluation. Receiver sensitivity at the FEC limit of $2 \cdot 10^{-3}$ is $1.5 \mathrm{dBm}$ and $4.3 \mathrm{dBm}$ for $60 \mathrm{~cm}$ and $1.3 \mathrm{~m}$ of wireless distance, respectively. Comb expansion reduces optical signal-to-noise ratio by 6.6-7.6 dB, as shown in Fig. 2(b), thus inducing a receiver sensitivity penalty of $5.9 \mathrm{~dB}$ and $5 \mathrm{~dB}$ for $60 \mathrm{~cm}$ and $1.3 \mathrm{~m}$ of wireless distance, respectively. BER is degraded for received optical power higher than $5 \mathrm{dBm}$ and $6 \mathrm{dBm}$ due to receiver saturation.

\section{Conclusion}

We have demonstrated bandwidth scalability, up to five sub-bands, of electrical 16QAM-OFDM signals in the $75-110 \mathrm{GHz}$ band employing optical comb generation. We have demonstrated optical and up to $1.3 \mathrm{~m}$ wireless transmission with a BER performance within FEC limits, showing the potential of multiband electrical OFDM in supporting future high-capacity hybrid optical-wireless applications approaching $50 \mathrm{~Gb} / \mathrm{s}$ and beyond. A 9.6-Gb/s OFDM signal (3.2-GHz RF bandwidth) has been transmitted over $22.8 \mathrm{~km}$ of SSMF and a comb-expanded OFDM signal up to $38.2 \mathrm{~Gb} / \mathrm{s}$ (14.4-GHz RF bandwidth) has been demonstrated for short-optical-distance applications. This work was supported in part by the FP7 ICT-249142 FIVER and FP7 ICT-224402 EURO-FOS Projects. The authors acknowledge the support from Tektronix, Agilent Technologies, Radiometer Physics GmbH, Rohde\&Schwarz, and $u^{2} t$ Photonics.

\section{References}

[1] A. Stöhr, "10 Gbit/s wireless transmission using millimeter-wave over optical fiber systems," in OFC 2011 , paper OTuO3.

[2] M. Beltrán, J. B. Jensen, X. Yu, R. Llorente, R. Rodes, M. Ortsiefer, C. Neumeyr, and I. Tafur Monroy, JSAC 29, 1295-1303 (2011).

[3] T. Nagatsuma, T. Takada, H.-J. Song, K. Ajito, N. Kukutsu, and Y. Kado, in IEEE Photonics Society 2010, pp. 385-386.

[4] F.-M. Kuo, C.-B. Huang, J.-W. Shi, N.-W. Chen, H.-P. Chuang, J. Bowers, and C.-L. Pan, IEEE Photon. J. 3, $209-219$ (2011).

[5] A. Kanno, K. Inagaki, I. Morohashi, T. Sakamoto, T. Kuri, I. Hosako, T. Kawanishi, Y. Yoshida, and K.-I. Kitayama, ELEX 8, 612-617 (2011).

[6] A. Kanno et al., "40 Gb/s W-band (75-110 GHz) 16-QAM radio-over-fiber signal generation and its wireless transmission," in ECOC 2011, We.10.P1.112.

[7] D. Zibar et al., "High-capacity wireless signal generation and demodulation in 75- to 110-GHz band employing all-optical OFDM,” PTL 23, 810-812 (2011).

[8]Z. Jiang, D. E. Leaird, and A. M. Weiner, “Optical processing based on spectral line-by-line pulse shaping on a phase-modulated CW laser,” JQE 42, 657-666 (2006).

[9] X. Liu and F. Buchali, "Intra-symbol frequency-domain averaging based channel estimation for coherent optical OFDM,” OE 16, 21944-21957 (2008). 